\title{
LOS SITIOS WEB COMO SERVICIOS DE INFORMACIÓN AL CIUDADANO: UN ESTUDIO SOBRE LOS 308 AYUNTAMIENTOS DE PORTUGAL ${ }^{1}$
}

\author{
Eduardo Alfredo Cardoso de Miranda* \\ Universidade Lusófona de Humanidades e Tecnologias. Lisboa. \\ Antonio Muñoz-Cañavate ${ }^{* *}$ \\ Facultad de Ciencias de la Documentación y la Comunicación. Universidad de Extremadura.
}

\begin{abstract}
Resumen: Las Administraciones Públicas han establecido desde la aparición de internet nuevas forma de comunicación, a través de los portales web, para gestionar la información que generan y que es de interés al ciudadano. Estos portales son parte de los sistemas de información de las Administraciones, en los que se gestiona tanto la información interior (back-office) como la información que se lanza al exterior (front-office), y han abierto un nuevo ámbito de trabajo para los profesionales de la información, y de investigación para el ámbito académico. El estudio que se presenta en este trabajo es resumen de una amplia tesis doctoral presentada en 2012 que ha analizado con detenimiento los servicios de los portales web en internet de los ayuntamientos de los 308 municipios de Portugal. Son los servicios de información, los servicios de comunicación y los servicios de transacción, aplicados a tres ámbitos: vida cotidiana, administración a distancia y participación política.

Palabras clave: Administración local; ayuntamientos; gestión de información; internet; e-gobierno; Portugal.
\end{abstract}

Title: THE WEBSITES AND INFORMATION SERVICES TO CITIZENS: A STUDY OF 308 MUNICIPALITIES IN PORTUGAL.

Abstract: Since the advent of the internet, Public Administrations have set up new ways in which to manage their information through their Web portals. These portals are part of an information system in which information is made available both internally (back-office) and externally (front-office). They have also meant that a new field has opened up for information professionals to work in and for academics to study.

The present communication is a synthesis of an extensive doctoral thesis presented in 2012 that analysed in detail the internet services of the Web portals of the councils of Portugal's 308 municipalities. In particular, these services were those of information, communication, and transactions applied to three areas: everyday life, distance administration, and political participation.

Keywords: Local government; municipal councils; information management; internet; e-government; Portugal.

\section{INTRODUCCIÓN}

Desde mediados de los años noventa del siglo XX las Administraciones Públicas se han beneficiado de la extensión de la aplicación más conocida de internet, el World Wide $W^{2} b^{2}$. A lo que hay que añadir el uso generalizado de la microinformática desde la década anterior. Todo ello ha contribuido a mejorar la eficacia y la eficiencia de estas organizaciones.

Sin embargo, aunque en principio pueda parecer que los cambios experimentados por las Administraciones se basan exclusivamente en la llegada de las TICs y de internet, en realidad esta transformación comienza varias décadas antes. Así, en el mundo occidental, con origen en Estados Unidos, se suceden desde mediados del siglo XX una serie de cambios que terminan extendiéndose en el sector público por el resto del mundo, a mayor o menor velocidad. En la década de los cincuenta y primeros sesenta se busca la manera de reestructurar la maquinaria de la Administración y sus problemas formales; a finales de los sesenta y primeros setenta se extiende la influencia americana de las políticas públicas; en los años setenta la crisis económica mundial y el recorte de presupuestos presiona al sector público a aplicar modelos de gestión del sector privado. Mientras, la implantación de la microinformática durante los años ochenta y de internet en los noventa sientan las bases del nuevo modelo organizativo.

Esta nueva dimensión de la Administración conocida como Administración electrónica ha dado lugar a toda una corriente de estudios y análisis en el ámbito académico y universitario. En los últimos años se han publicado numerosos trabajos sobre la administración electrónica realizados bajo diferentes perspectivas. Se han llevado a cabo estudios globales acerca de la incidencia y el impacto de la Administración electrónica en el conjunto de un país

\footnotetext{
*1sociologia@gmail.com

**amunoz@alcazaba.unex.es
}

Recibido: 19-11-2014; aceptado: 17-03-2015.

CARDOSO DE MIRANDA, E.A. y MUÑOZ CAÑAVATE, A. Los sitios web como servicios de información al ciudadano: un estudio sobre los 308 ayuntamientos de Portugal. Anales de Documentación, 2015, vol. 18, $\mathrm{n}^{\circ} 1$. Disponible en: http://dx.doi.org/10.6018/analesdoc.18.1.212681 
(Allen, 2001; Holliday, 2005; Chen, 2009; Chan, 2008; Groznik, 2009; Muñoz-Cañavate, 2011). Se han analizado las diferentes etapas y desarrollos derivados de este nuevo paradigma (Layne, 2000; Gil-García, 2007). Se han estudiado los costos de aplicación (Bertot, 2008), los modelos de Administración electrónica (Coursey, 2008), las implicaciones laborales que para los profesionales de las ciencias de la información puede tener la administración electrónica (Chain, 2008), o realizado estudios acerca de los websites de las administraciones locales (Armstrong, 2011; Duarte, 2000; Shi, 2007) entre otros aspectos.

De este nuevo enfoque también participan las leyes de transparencia en los gobiernos que posibilitan que los ciudadanos puedan solicitar información sobre los asuntos más variados.

\section{EL PROCESO DE CAMBIO EN PORTUGAL}

Las Administraciones Públicas de Portugal comienzan en las dos últimas décadas del siglo XX, al igual que en otros países, a utilizar las tecnologías de la información y de las comunicaciones (TICs) con el propósito de optimizar su modelo de trabajo y con un objetivo general: mejorar los servicios prestados a ciudadanos y empresas.

La entrada de Portugal en la Comunidad Europea en 1986 supone un fuerte revulsivo a todas sus políticas, y permite a este país implicarse en los distintos proyectos europeos que tienen como objetivo impulsar las TICs como catalizadoras de una nueva sociedad. Así, desde los años noventa del siglo XX en Europa se lanzan varias iniciativas políticas de gran calado. En 1993 se publica el Plan Delors o Libro Blanco basado en el uso de las tecnologías de la información para conseguir mayor crecimiento, competitividad y empleo. Le sigue el Informe Bangemann (Europa y la sociedad global de la información) que define diez ámbitos para concretar las políticas de sociedad de la información. ${ }^{3}$ A aquellos documentos iniciales le suceden otros como el Plan de Acción de la Comisión de 1994 y diversos grupos creados ad hoc para el análisis de todas estas medidas, como el Grupo de Alto Nivel en 1995 que debate acerca de los aspectos sociales que implica el uso de las TICs en la vida de los europeos; o el Forum Europeo de la Sociedad de la Información, también en 1995, formado por 128 miembros con un grupo específico orientado a los servicios públicos que recomienda introducir masivamente las tecnologías de la información para acercar la Administración a los ciudadanos (Muñoz-Cañavate, 2003, p. 68-69).

Desde esos momentos en Europa aparecen una serie de planes plurianuales dedicados a acelerar las estrategias políticas destinadas a impulsar la sociedad de la información: eEurope 2002, eEurope 2005 y eEurope 2010. Y organismos como la OCDE lanzan iniciativas políticas como la desarrollada en el documento de 2003 "The eGovernment Imperative" (OCDE, 2003).

Como consecuencia del entorno europeo ya descrito, en Portugal en la segunda mitad de los años noventa se comienzan a sentar las bases políticas para cambiar los métodos y herramientas usadas en la Administración Pública. Anabela Pedroso, de la Agência para a Sociedade do Conhecimento (UMIC), señala que en Portugal arrancan en 1996 una serie de estudios que tienen como finalidad conocer la situación en la que se encuentra la Administración portuguesa (Pedroso, 2006). Los resultados no son muy buenos ya que se descubre una Administración que trabaja para sí misma, con duplicidad de funciones dentro de los propios servicios, además de una falta de articulación y de comunicación entre los diferentes organismos públicos que, según los estudios, funcionan en circuito cerrado. Entre estos análisis se realizan encuestas a los ciudadanos. Algunas de las conclusiones reflejan la situación de la Administración en Portugal. Por ejemplo, los ciudadanos pedían a la Administración que les dieran una misma respuesta independientemente del organismo administrativo al que preguntasen. Una frase de un ciudadano define la Administración portuguesa en esa fecha: "La Administración Pública es la organización que menos contribuye a mi felicidad”. Este diagnóstico hace que el gobierno portugués tenga que repensar la Administración buscando en el apoyo de las TICs una Administración centrada en el ciudadano y en base a cuatro ejes: a) una oferta de servicios, b) la forma en la que se prestan esos servicios y los medios a través de los cuales se prestan, c) el papel de las TICs (con especial incidencia en la identidad digital, la gestión de la información y las infraestructuras), y d) una aproximación empresarial a la gestión de los servicios (Muñoz-Cañavate, 2006, p. 73).

Para la materialización de estas políticas y directrices tiene una gran importancia, en la segunda mitad de la década de los noventa, el Ministerio de Ciencia y Tecnología y las políticas de sociedad de la información definidas a través del Libro Verde de la Sociedad de la Información (1997) que en su aplicación a los ayuntamientos portugueses señala textualmente: "El papel desempeñado por las autoridades locales a través de iniciativas que conduzcan a la creación de redes electrónicas municipales, gestionadas en colaboración con las organizaciones que representan los intereses locales, crea nuevas formas de expresión ciudadana y es un medio eficaz para la democratización del acceso a las 
nuevas formas de información digital, así como para promover el enriquecimiento de esta última con contenidos de interés local".

Este documento continua diciendo: "La difusión de información a los ciudadanos y las empresas siempre debe estar disponible por medios electrónicos. De ahí la necesidad de una revisión a fondo de cómo se dirige la administración a los ciudadanos y las empresas. [...] Es en este sentido que entendemos el movimiento acelerado observado en la sociedad portuguesa para la disponibilidad de páginas world wide web en internet, de forma similar a lo que ha ocurrido en los países en los que la sociedad de la información está en una etapa superior de desarrollo a la nuestra".

En aquella etapa otros hitos importantes son la creación en 1994 de SAPO (Servidor de Apontadores Portugueses Online), o en 1997 de la Rede Ciência Tecnologia e Sociedade para a Computação Científica (RCTS). Otros organismos permiten poner en marcha las políticas diseñadas por los distintos gobiernos portugueses: la Agência para a Modernização Administrativa (AMA), la Agência para a Sociedade do Conhecimento (UMIC) y el Instituto Nacional para a Reabilitação e Centro de Gestão da Rede Informática do Governo (CEGER).

Algunas aplicaciones de gran éxito para la transformación de la Administración portuguesa han sido el programa Simplex, que engloba un conjunto de iniciativas tendentes a la simplificación de la vida administrativa; el documento de identidad electrónico (cartão de cidadão); o el portaldocidadao.pt, como un portal de acceso único a la información de la administración pública del país.

Por otra parte, distintos informes sobre administración electrónica describen la situación de Portugal en el ámbito internacional. Los dos indicadores más importantes son el de Naciones Unidas a través del "UN eGovernment Survey" y el análisis que realiza la Unión Europea (UE), a través de la consultora internacional Capigemini.

En el índice de Naciones Unidas, Portugal se encontraba en 2005 en el puesto 30 del ranking mundial, en 2008 en el puesto 31, en 2010 en el puesto 39, mientras que en el último estudio de 2012 ha subido al puesto 33 de 190 países. Lo cual sitúa a Portugal en el grupo de países más avanzados. Estos datos, por otra parte, se encuentran en la línea de los informes de la Comisión Europea que analizan, en el contexto de la administración electrónica, la existencia o no de los servicios públicos básicos electrónicos disponibles en internet para cada país de la Unión Europa. Los informes señalan que Portugal tenía en 2010 el 100\% de los servicios disponibles para sus ciudadanos, al mismo nivel que Austria, Irlanda, Italia, Malta y Suecia.

Pero para completar el análisis de Portugal a través de los informes y estadísticas internacionales hay que reflejar el uso que los ciudadanos hacen de estos servicios. Así, la Oficina Europea de Estadística (Eurostat) recoge desde hace años para todos los países de la UE tres indicadores que reflejan el uso que los ciudadanos europeos hacen de los servicios de administración electrónica de cada país: a) obtención de información de las autoridades públicas, b) descarga de formularios y c) envío de formularios (tanto para ciudadanos como para empresas). Los datos son muy reveladores si comparamos las cifras de 2009 con las de 2012. Así mientras en 2009 sólo un 18\% de los portugueses obtenía información de las autoridades públicas, en 2012 la cifra se elevaba al 58\%. En 2009 sólo un 14\% de ciudadanos descargaba formularios, dato que subía al 36\% en 2012. Finalmente el proceso más complejo, el envío de formularios era utilizado por un 16,3\% de portugueses en 2009 y por un $43 \%$ en 2012. Las cifras para las empresas presentan cifras mucho más elevadas para todos los años.

Finalmente, la legislación portuguesa también se ha actualizado siguiendo las directivas europeas, como el acceso a los documentos administrativos y su reutilización (Lei $n^{\circ}$ 46/2007); compras públicas electrónicas (Decreto-Lei $n^{\circ}$ 18/2008); comercio electrónico (Decreto-Lei $n^{0} 7 / 2004$ ); firmas electrónicas (Decreto-Lei $n^{\circ}$ 66/2003, Decreto-Lei $n^{\circ}$ 165/2004, y Decreto-Lei $\left.n^{\circ} 116 \mathrm{~A} / 2006\right)$; sistema de certificación electrónica del Estado portugués (Decreto-Lei $n^{\circ}$ 116A/2006); facturas electrónicas (Decreto-Lei $n^{\circ}$ 256/2003, y Resolución del Consejo de Ministros 137/2005); privacidad en las comunicaciones electrónicas (Decreto-Lei ${ }^{\circ}$ 41/2004). Respecto a la normativa sobre transparencia y buen gobierno, en diciembre de 2011 se presentó al parlamento portugués el proyecto de ley 115/XII (Lei da Transparência Activa da Informação Pública), pero no fue aprobado. De manera que en 2015 Portugal todavía no disponía de una norma similar a la aprobada en otros países europeos.

\section{EL OBJETO DE ESTUDIO}

Este trabajo se ha centrado en la Administración local portuguesa, que se compone de 308 cámaras municipales o ayuntamientos. Estos ayuntamientos quedan enmarcados en la división administrativa de Portugal que fue definida en 
1964 a través del Decreto-Lei n. 46139/1964 y que incluye a los municipios en distritos. Así, en la actualidad existen 18 distritos en el Portugal continental (Aveiro, Beja, Braga, Bragança, Castelo Branco, Coímbra, Évora, Faro, Guarda, Leiria, Lisboa, Portalegre, Porto, Santarém, Setúbal, Viana do Castelo, Vila Real y Viseu) y dos regiones autónomas insulares (Región autónoma de las Azores/Angra Heroísmo y Región autónoma de Madeira/Funchal).

El estudio de Duarte y Martins do Amaral en 2000 para los ayuntamientos portugueses nos dice que en dicho año sólo 153 de las 308 cámaras municipales disponían de sitio web oficial, es decir el 49\% de los municipios. En la fecha de realización de este estudio (segundo semestre de 2009 y primer semestre de 2010) el 100\% de los ayuntamientos de Portugal tiene sitio web oficial.

\begin{tabular}{|l|l|l|}
\hline & Número & Porcentaje \\
\hline Aveiro & 19 & 6,2 \\
\hline Beja & 14 & 4,5 \\
\hline Braga & 14 & 4,5 \\
\hline Bragança & 12 & 3,9 \\
\hline Castelo Branco & 11 & 3,6 \\
\hline Coimbra & 17 & 5,5 \\
\hline Évora & 14 & 4,5 \\
\hline Faro & 16 & 5,2 \\
\hline Guarda & 14 & 4,5 \\
\hline Leiria & 16 & 5,2 \\
\hline Lisboa & 16 & 5,2 \\
\hline Portalegre & 15 & 4,9 \\
\hline Porto & 18 & 5,8 \\
\hline Santarém & 21 & 6,8 \\
\hline Setúbal & 13 & 4,2 \\
\hline Vianado Castelo & 10 & 3,2 \\
\hline Vila Real & 14 & 4,5 \\
\hline Viseu & 24 & 7,8 \\
\hline Angra Heroísmo & 19 & 6,2 \\
\hline Funchal & 11 & 3,6 \\
\hline Total & 308 & 100 \\
\hline
\end{tabular}

Tabla I. Número de ayuntamientos por distritos.

Es necesario señalar que estos ayuntamientos disponen de una cierta autonomía conseguida tras la revolución de 1974, y el advenimiento de la democracia que altera todos los niveles administrativos. Así, el gobierno experimenta una descentralización política que afecta sobre todo al ámbito local. Esta política de descentralización elimina el papel secundario que había tenido el poder local durante la dictadura del denominado Estado Novo. La fase constitucional del poder local se inicia con la aprobación de la Constitución en abril de 1976 y la celebración de las primeras elecciones municipales en diciembre de 1976 (Branco, 1998, p. 17). Desde ese instante la administración local portuguesa asume una responsabilidad cada vez mayor basada en la descentralización económica y política, que se refleja en la existencia de un microcosmos de poder basado en espacios de democracia representativa. Macedo e Sousa (2000) resume muy bien en que se ha convertido el ámbito local: "Una de las formas más interesantes de acercarse a una administración local es considerarla a escala como un pequeño gobierno. [...] Todo está ahí: medio ambiente, cultura, deportes, obras públicas y finanzas, entre otros. [...] Incluso en las zonas donde el marco de la intervención de las autoridades locales es más difuso como en trabajo, o economía, allí también, el grado y las expectativas de acción de las autoridades locales son cada vez mayores”.

El estudio que aquí se presenta ha girado en torno a la gestión de la información corporativa de los 308 ayuntamientos portugueses en internet a través de sus portales corporativos en la red. El universo de estudio ha tomado la totalidad de los municipios portugueses, tanto del Portugal continental como del Portugal insular. El trabajo se extendió durante varios años desde su planificación en 2008 hasta su presentación en 2012.

\section{METODOLOGÍA}

El proyecto original se centró en dos ejes. Por un lado los contenidos de los portales de los ayuntamientos o cámaras municipales portuguesas, y por otro el análisis de los recursos humanos que estructuran las plantillas 
dedicadas a gestionar la información. Este artículo recoge únicamente los resultados del primer estudio: los contenidos y servicios de información.

El trabajo parte del conocimiento previo de los estudios realizados a la Administración local en España, tanto en su evolución histórica (Muñoz-Cañavate, 2012) como en las investigaciones sobre la gestión de los contenidos y personal dedicado a su tratamiento (Chain, 2008b). De tal manera que este estudio aplicado a Portugal es heredero de aquellos.

Las direcciones web y de email de los 308 municipios se obtuvieron del sitio web de la Asociación Nacional de Municipios Portugueses (http://www.anmp.pt).

El examen de los contenidos y servicios definidos en los portales web describe los dos ámbitos que coexisten en una administración pública. Por un lado la dimensión política definida por personas que tras un proceso democrático llegan a las estructuras de poder de las administraciones, y por otro la dimensión más administrativa formada por el personal de estas organizaciones que coexiste con la esfera política. Tanto una dimensión como otra son importantes y se ven totalmente envueltas en la reingeniería de procesos que conlleva la administración electrónica.

Estas dos dimensiones (política y administrativa) quedan recogidas en el cuestionario aplicado por el método de observación directa a los contenidos de los portales web, que aparece en Anexo. Este cuestionario ha tenido como esquema inicial el señalado en el documento de la Comisión Europea La información del sector público: un recurso clave para Europa [COM (1998) 585], también conocido como Libro Verde sobre la información del sector público en la sociedad de la información. En dicho documento se estructuran los servicios en tres tipos: a) servicios de información, b) servicios de comunicación y c) servicios de transacción. En estos tres niveles de relaciones se puede observar el nivel de interacción entre las Administraciones con el exterior. El nivel informativo usa un nivel de relación unilateral, el nivel comunicativo imprime una relación bilateral al producirse una comunicación, mientras que el nivel transaccional puede implicar un nivel mayor de contacto, que en algunos casos conlleva la participación directa del ciudadano en el proceso decisorio.

A su vez cada uno de estos servicios se ha aplicado a tres ámbitos generales: a) vida cotidiana, b) administración a distancia y c) participación política.

La combinación de los tres servicios con los tres ámbitos ha posibilitado un esquema de nueve espacios de estudio como el que presenta la Tabla II, en cuyo interior aparecen algunos ejemplos de los ítems utilizados en el cuestionario y que da idea del contenido aplicado en cada uno de los nueve sectores analizados.

Duarte y Martins do Amaral analizan en el año 2000 los contenidos de los websites municipales a través de tres ejes: a) servicios de información genérica del municipio, que definen como la información de interés general; b) información del poder municipal, que incluye los contenidos de la vida diaria del ayuntamiento, reglamentos, decisiones políticas, eventos; y finalmente, c) los servicios de información online, que incluyen los contenidos que exigen interacción entre los ciudadanos y el poder local, y que se realizan a través del envío de formularios o mensajes electrónicos, y que por tanto exige más exigencias desde el punto de vista tecnológico (Duarte, 2000, p. 19-20).

\begin{tabular}{|l|l|l|l|}
\hline & Servicios de información & $\begin{array}{l}\text { Servicios de } \\
\text { comunicación }\end{array}$ & $\begin{array}{l}\text { Servicios de } \\
\text { transacción }\end{array}$ \\
\hline Vida cotidiana & $\begin{array}{l}\text { - Información laboral, doméstica, sobre } \\
\text { educación, salud, cultura, transportes, } \\
\text { medio ambiente, etc. }\end{array}$ & $\begin{array}{l}\text { - Debates sobre } \\
\text { cuestiones de la vida } \\
\text { cotidiana. } \\
\text { - Tablón de anuncios de } \\
\text { tipo laboral o doméstico. }\end{array}$ & $\begin{array}{l}\text { - Reserva de } \\
\text { billetes, } \\
\text { matriculación } \\
\text { en cursos. }\end{array}$ \\
\hline $\begin{array}{l}\text { Administración } \\
\text { a distancia }\end{array}$ & $\begin{array}{l}\text { - Direcciones de servicios públicos. } \\
\text { - Guía de procedimientos } \\
\text { administrativos. } \\
\text { - Registros y bases de datos públicos. }\end{array}$ & $\begin{array}{l}\text { - Contacto por correo } \\
\text { electrónico con } \\
\text { funcionarios. }\end{array}$ & $\begin{array}{l}\text { - Presentación } \\
\text { electrónica de } \\
\text { formularios. }\end{array}$ \\
\hline $\begin{array}{l}\text { Participación } \\
\text { política }\end{array}$ & $\begin{array}{l}\text { - Leyes, documentos parlamentarios, } \\
\text { programas políticos, documentos de } \\
\text { consulta. } \\
\text { - Información previa sobre el proceso } \\
\text { decisorio. }\end{array}$ & $\begin{array}{l}\text { - Debates sobre } \\
\text { problemas políticos. } \\
\text { - Contacto por correo } \\
\text { electrónico con políticos. }\end{array}$ & $\begin{array}{l}\text { - Referendos. } \\
\text { - Slecciones. }\end{array}$ \\
\hline
\end{tabular}

Tabla II. Esquema aplicado a los contenidos de los portales de los ayuntamientos. 
El lector puede observar en el Anexo y en las distintas tablas del apartado de resultados la exhaustividad del estudio. En total se han recogido 59 indicadores por cada uno de los 308 ayuntamientos, lo que ha permitido recopilar un total de 18.172 datos en el periodo de estudio. Este procedimiento metodológico y el sistema de recogida (la observación directa) han posibilitado la obtención del 100\% de la información requerida en un principio, en tanto que su análisis ha dependido en exclusiva del trabajo de los investigadores aplicado directamente a las sedes web.

Para el conocimiento de cada sitio web se ha utilizado un sistema de puntuación aplicado a cada uno de los indicadores. Así se ha puntuado de 0 a 1 ( 0 cuando no existe información y 1 cuando sí existe), o de 0 a 2 ( 0 cuando no existe información, 1 cuando sí existe información pero sin actualizar y 2 cuando existe información actualizada). El tratamiento posterior se ha realizado con el software SPSS.

De los nueve ámbitos a dos se ha aplicado la puntuación 0-1-2. Han sido: a) servicios de información/vida cotidiana y b) servicios de información/participación política, ya que son dos ámbitos susceptibles de disponer de información sin actualizar. A los otros siete ámbitos se les ha aplicado la puntuación 0-1.

La recogida de datos de los portales web de los 308 ayuntamientos de Portugal se realiza entre el segundo semestre de 2009 y el primero de 2010.

\section{RESULTADOS}

\subsection{El estudio de los contenidos}

Las tablas que aparecen a continuación representan el peso total en porcentaje de los 308 ayuntamientos. Para su mejor comprensión se incluyen los resultados totales y los comentarios para las nueve áreas. Todo ello estructurado en cuatro apartados: ámbito general de vida cotidiana (5.1.1), ámbito general de administración a distancia (5.1.2), ámbito general de participación política (5.1.3) y resultados globales (5.1.4). Este último apartado recoge el porcentaje global de todos los indicadores unidos dentro de cada uno de los nueve espacios estudiados, con los datos de los distritos portugueses con mejores resultados y el distrito con peor resultado.

\subsubsection{Vida cotidiana}

La Tabla III reúne los resultados de los servicios de información aplicados al ámbito general de la vida cotidiana. Este nivel de relaciones es unilateral por parte de la Administración y vuelca aquella información que puede interesar a un ciudadano en su vida cotidiana en una población. Por tanto, tal y como se puede ver en la tabla, recoge aspectos muy variados como información sobre salud, educación, deporte, cultura, servicios sociales, transportes, urbanismo, seguridad y datos estadísticos y comerciales. Los datos reflejan un elevado nivel de información en todas las áreas evaluadas con información actualizada. La excepción con valores más bajos es para los servicios y para transportes, si bien en este caso hay grandes diferencias entre ciudades ya que no todos los municipios utilizan todos los medios de transporte, aunque la información sobre los horarios es alta (86,4\%). 


\begin{tabular}{|c|c|c|c|}
\hline & Existe & No existe & $\begin{array}{c}\text { Sin } \\
\text { actualizar }\end{array}$ \\
\hline \multicolumn{4}{|l|}{ Información general } \\
\hline - Datos postales de la ciudad & 78,2 & 21,8 & 0,0 \\
\hline - Información de la historia de la ciudad & 80,8 & 19,2 & 0,0 \\
\hline - Información turística (monumentos) & 99,4 & 0,6 & 0,0 \\
\hline - Teléfonos de interés del Municipio & 91,9 & 8,1 & 0,0 \\
\hline \multicolumn{4}{|l|}{ Información sobre salud pública } \\
\hline - Sistema sanitario (instalaciones) & 90,9 & 9,1 & 0,0 \\
\hline - Horario de las farmacias de guardia & 99,4 & 0,6 & 0,0 \\
\hline \multicolumn{4}{|l|}{ Información sobre educación } \\
\hline - Instalaciones & 100 & 0,0 & 0,0 \\
\hline - Oferta escolar & 97,4 & 2,6 & 0,0 \\
\hline - Bolsas de estudio & 98,1 & 1,9 & 0,0 \\
\hline \multicolumn{4}{|l|}{ Información sobre deporte } \\
\hline - Instalaciones deportivas & 94,2 & 5,8 & 0,0 \\
\hline - Eventos deportivos (agenda) & 95,5 & 1,6 & 2,9 \\
\hline \multicolumn{4}{|l|}{ Información sobre cultura } \\
\hline - Museos & 94,5 & 5,5 & 0,0 \\
\hline - Biblioteca municipal & 62,7 & 37,3 & 0,0 \\
\hline - Otras bibliotecas & 51,0 & 47,7 & 1,3 \\
\hline - Eventos culturales & 84,4 & 14,0 & 1,6 \\
\hline - Consulta online & 66,2 & 33,8 & 0,0 \\
\hline \multicolumn{4}{|l|}{ Información sobre servicios sociales } \\
\hline - Instalaciones para mujeres & 8,1 & 91,9 & 0,0 \\
\hline - Instalaciones para jóvenes & 7,5 & 91,9 & 0,6 \\
\hline - Instalaciones para inmigrantes & 1,6 & 98,4 & 0,0 \\
\hline \multicolumn{4}{|l|}{ Información sobre transportes } \\
\hline - Autobuses & 99,7 & 0,3 & 0,0 \\
\hline - Metro & 10,1 & 89,9 & 0,0 \\
\hline - Avión & 10,1 & 89,9 & 0,0 \\
\hline - Tren & 8,1 & 91,9 & 0,0 \\
\hline - Marítimo & 0,6 & 99,4 & 0,0 \\
\hline - Horarios & 86,4 & 13,6 & 0,0 \\
\hline \multicolumn{4}{|l|}{ Información urbana } \\
\hline - Mapas de la ciudad & 100,0 & 0,0 & 0,0 \\
\hline - Mapas interactivos de la ciudad & 91,2 & 8,8 & 0,0 \\
\hline \multicolumn{4}{|l|}{ Información estadística } \\
\hline - Sobre la población & 98,1 & 1,9 & 0,0 \\
\hline - Sobre datos económicos & 0,0 & 100,0 & 0,0 \\
\hline \multicolumn{4}{|l|}{ Información comercial } \\
\hline - Sobre mercados & 98,4 & 1,6 & 0,0 \\
\hline - Sobre comercio & 99,0 & 1,0 & 0,0 \\
\hline \multicolumn{4}{|l|}{ Información sobre seguridad } \\
\hline - Policía (contactos) & 92,9 & 7,1 & 0,0 \\
\hline - Bomberos (contactos) & 97,1 & 2,9 & 0,0 \\
\hline - Protección civil (contactos) & 34,4 & 65,6 & 0,0 \\
\hline
\end{tabular}

Tabla III. Servicios de información. Vida cotidiana. Valor medio en \%.

La Tabla IV recoge los resultados del nivel de interactividad o comunicación bidireccional entre los ciudadanos y empresas con la Administración Pública. Es necesario señalar que en las fechas de realización de este estudio no existía la diversidad de medios sociales que encontramos en la actualidad ${ }^{4}$ (redes sociales, marcadores, blog, microblogging, etc.). En este análisis se incluyeron a las listas de correo electrónico consideradas como uno de los primeros medios sociales existentes. En general los resultados son aceptables tanto en el uso de listas de correo como de foros. 


\begin{tabular}{|l|l|l|}
\hline Foros de discusión & Existe & No existe \\
\hline - Lista de correo electrónico & 82,8 & 17,2 \\
\hline - A través de foros & 69,2 & 30,8 \\
\hline - A través de chat & 42,9 & 57,1 \\
\hline
\end{tabular}

Tabla IV. Servicios de comunicación. Vida cotidiana. Valor medio en \%.

Por otra parte, la tabla 5 presenta los resultados del aspecto que supone un mayor nivel de implicación tecnológica y administrativa, es decir la posibilidad de realizar compras de entradas en eventos cotidianos como teatro o eventos deportivos. Aunque en este caso se buscaba tanto la existencia de ese mecanismo gestionado por el propio ayuntamiento como la existencia del servicio externalizado, es decir a través de portales privados de venta de entradas.

\begin{tabular}{|l|l|l|}
\hline Reserva o compra de billetes & Existe & No existe \\
\hline - Teatro municipal & 57,8 & 42,2 \\
\hline - Eventos deportivos & 83,1 & 16,9 \\
\hline
\end{tabular}

Tabla V. Servicios de transacción. Vida cotidiana. Valor medio en \%.

Los datos de este primer bloque, dedicado a aspectos de la vida cotidiana, presenta unos resultados elevados sobre los indicadores evaluados, tanto en los servicios de información, como en los servicios de comunicación (que implica una interacción con el exterior) y los servicios de transacción centrados en la reserva o compra de billetes para actividades culturales y deportivas.

\subsubsection{Administración a distancia}

Este apartado dedicado a la administración a distancia recoge aquellos ítems que definen de manera más clara el proceso de inserción de los ayuntamientos portugueses en la administración electrónica. Así, en este apartado se presenta, en el contexto de los servicios de información (Tabla VI), aquella información que más puede interesar al ciudadano en sus relaciones con la institución municipal (directorios, guías de trámites, etc.). Es necesario destacar el elevado porcentaje (en varios casos el 100\% de los ayuntamientos) de ítems en los que sí existe la información requerida.

\begin{tabular}{|l|r|c|}
\hline $\begin{array}{l}\text { Directorio de los servicios del } \\
\text { ayuntamiento }\end{array}$ & Existe & No existe \\
\hline - Teléfono & 100,0 & 0,0 \\
\hline - Correo electrónico & 100,0 & 0,0 \\
\hline - Correo postal & 100,0 & 0,0 \\
\hline Trámites administrativos & & \\
\hline - Guía de trámites administrativos & 97,4 & 2,6 \\
\hline - Ordenanzas fiscales & 92,5 & 7,5 \\
\hline - Ordenanzas municipales & 100,0 & 0,0 \\
\hline Servicios de prensa & & 0,0 \\
\hline - Comunicados & 100,0 & 0,0 \\
\hline - Agenda de los actos del alcalde & 100,0 & \\
\hline Asamblea municipal & 100,0 & 0,0 \\
\hline - Nombre del alcalde & 69,5 & 30,5 \\
\hline - Correo electrónico del alcalde & 100,0 & 0,0 \\
\hline - Nombre de los concejales & 79,9 & 20,1 \\
\hline - Correo electrónico de los concejales & & \\
\hline
\end{tabular}

Tabla VI. Servicios de información. Administración a distancia. Valor medio en \%.

Sobre los servicios de comunicación (Tabla VII) el estudio releva un menor desarrollo en el tiempo de elaboración del estudio del contacto electrónico con los distintos departamentos en los que se desarrolla el trabajo administrativo a través del personal de los ayuntamientos. Lo que contrasta con la Tabla VIII (servicios de transacción) que define un elevado nivel de procedimientos que los ciudadanos y empresas sí pueden tramitar a través de internet. 


\begin{tabular}{|c|c|c|}
\hline $\begin{array}{c}\text { Contacto por correo electrónico con } \\
\text { los funcionarios del ayuntamiento }\end{array}$ & Existe & No existe \\
\hline $\begin{array}{c}\text { - Lista de todos los servicios } \\
\text { municipales }\end{array}$ & 33,1 & 66,9 \\
\hline - Lista de las áreas de gobierno & 18,8 & 81,2 \\
\hline
\end{tabular}

Tabla VII. Servicios de comunicación (interacción con los ciudadanos). Administración a distancia. Valor medio en \%.

\begin{tabular}{|c|c|c|}
\hline $\begin{array}{c}\text { Presentación electrónica } \\
\text { documentos/solicitudes/trámites }\end{array}$ & Existe & No existe \\
\hline Población & & \\
\hline - Recepción de documentos & 94,2 & 5,8 \\
\hline Educación & & 15,9 \\
\hline - Recepción de documentos & 84,1 & 15,3 \\
\hline Territorio, urbanismo y obras & & 33,1 \\
\hline $\begin{array}{c}\text { - Trámites de expedientes de } \\
\text { obras }\end{array}$ & 84,7 & 30,5 \\
\hline $\begin{array}{c}\text { - Consulta de expedientes } \\
\text { Circulación, vehículos ysportes }\end{array}$ & 66,9 & 10,1 \\
\hline - Pago de impuesto de vehículos & 69,5 & 3,9 \\
\hline $\begin{array}{c}\text { Comercio, industria y consumo } \\
\text { - Trámites sobre la apertura de }\end{array}$ & 89,9 & \\
\hline $\begin{array}{c}\text { establecimientos Estado del expediente sobre } \\
\text { licencias/actividad }\end{array}$ & 96,1 & \\
\hline
\end{tabular}

Tabla VIII. Servicios de transacción. Administración a distancia. Valor medio en \%.

\subsubsection{Participación política}

Las Tablas IX (servicios de información), X (servicios de comunicación) y XI (transacción) relacionan el uso de las tecnologías de la información con la denominada democracia electrónica en este ámbito de la participación política. Es necesario señalar (tal y como se ha explicado en el apartado de vida cotidiana), que en el tiempo de realización del estudio apenas estaban desarrollados los nuevos medios sociales, exceptuando los foros de discusión. Son aspectos que se habrían incorporado a un estudio actualizado (como la existencia de ayuntamientos con perfiles en, por ejemplo, Facebook o Twitter, al igual que el uso de estos medios por parte de los cuadros políticos).

La Tabla IX presenta valores muy elevados (100\%) lo que significa que la totalidad de los ayuntamientos vuelca en sus portales la información que generan los órganos de decisión municipales (actas de plenos, boletines municipales de información y decretos del alcalde). Un aspecto vital de la transparencia informativa de las instituciones municipales.

Sin embargo, aunque la fase informativa de este ámbito (el de la democracia electrónica) esté más desarrollada contrasta con la fase comunicativa (Tabla X) y la escasez de debates, y la inexistencia de retransmisiones de los plenos por internet. Si bien, tal y como se indica en la Tabla VI, la mayor parte de los alcaldes y concejales habían hecho pública su dirección de correo electrónico. Finalmente la Tabla XI refleja el más alto nivel de desarrollo de la edemocracia que implica el uso de las TICs para que los ciudadanos participen del proceso decisorio. El dato es evidente $(0 \%)$ y manifiesta una situación que solo puede mejorar.

\begin{tabular}{|l|c|c|c|}
\hline & Existe & No existe & No actualizada \\
\hline Documentos & & & 0,0 \\
\hline - Actas de plenos & 99,7 & 0,3 & 0,0 \\
\hline - Boletín municipal de información & 100,0 & 0,0 & 0,0 \\
\hline $\begin{array}{l}\text { - Decretos y/o despachos del } \\
\text { alcalde }\end{array}$ & 100,0 & 0,0 & \\
\hline
\end{tabular}

Tabla IX. Servicios de información. Participación política. Valor medio en \%. 


\begin{tabular}{|l|c|c|}
\hline & Existe & No existe \\
\hline Debates de problemas políticos & & \\
\hline - Debates & 12,7 & 87,3 \\
\hline Retransmisión de los plenos & & \\
\hline - Por TV & 0,0 & 100,0 \\
\hline - Por voz & 0,0 & 100,0 \\
\hline
\end{tabular}

Tabla X. Servicios de comunicación. Participación política. Valor medio en \%.

\begin{tabular}{|l|c|c|}
\hline & Existe & No existe \\
\hline Votación por internet & & \\
\hline - Votación & 0,0 & 100,0 \\
\hline
\end{tabular}

Tabla XI. Servicios de transacción. Participación política. Valor medio en \%.

\subsubsection{Resultados globales}

Los resultados de la Tabla XII se corresponden con el resumen del total de los 308 portales de los ayuntamientos analizados en los tres ámbitos generales (vida cotidiana, administración a distancia y participación política) sobre los tres tipos de servicios (información, comunicación y transacción).

\begin{tabular}{|c|c|c|c|c|c|c|c|}
\hline \multirow{3}{*}{ Vida cotidiana } & \multicolumn{3}{|c|}{ Servicios de información } & \multicolumn{2}{|c|}{$\begin{array}{cc}\text { Servicios } & \text { de } \\
\text { comunicación }\end{array}$} & \multicolumn{2}{|c|}{$\begin{array}{cc}\text { Servicios } & \text { de } \\
\text { transacción }\end{array}$} \\
\hline & Existe & $\begin{array}{c}\text { Sin } \\
\text { actualizar }\end{array}$ & $\begin{array}{l}\text { No } \\
\text { existe }\end{array}$ & Existe & $\begin{array}{l}\text { No } \\
\text { existe }\end{array}$ & Existe & $\begin{array}{l}\text { No } \\
\text { existe }\end{array}$ \\
\hline & $68,47 \%$ & $0,19 \%$ & $31,34 \%$ & $64,97 \%$ & $35,03 \%$ & $70,45 \%$ & $29,55 \%$ \\
\hline $\begin{array}{l}\text { Administración a } \\
\text { distancia }\end{array}$ & Existe & & o existe & Existe & $\begin{array}{l}\text { No } \\
\text { existe }\end{array}$ & Existe & $\begin{array}{l}\text { No } \\
\text { existe }\end{array}$ \\
\hline & $94,94 \%$ & & $06 \%$ & $25,95 \%$ & $74,05 \%$ & $83,63 \%$ & $16,37 \%$ \\
\hline $\begin{array}{l}\text { Participación } \\
\text { política }\end{array}$ & Existe & $\begin{array}{c}\text { Sin } \\
\text { actualizar }\end{array}$ & $\begin{array}{l}\text { No } \\
\text { existe }\end{array}$ & Existe & $\begin{array}{l}\text { No } \\
\text { existe }\end{array}$ & Existe & $\begin{array}{l}\text { No } \\
\text { existe }\end{array}$ \\
\hline & $99,9 \%$ & $0,1 \%$ & $0,00 \%$ & $11,3 \%$ & $88,7 \%$ & $0,00 \%$ & $100 \%$ \\
\hline
\end{tabular}

Tabla XII. Peso medio porcentual de cada uno los servicios.

\subsubsection{Vida cotidiana}

En servicios de información en el ámbito de vida cotidiana, nos encontramos con un valor porcentual total del $68,4 \%$ en contenidos existentes y actualizados para el conjunto de Portugal. Por distritos, el valor medio porcentual más alto para estos contenidos actualizados ha sido para Evora (78,99\%), seguido de Lisboa (70,96\%), y el valor más bajo ha sido para el distrito de Viana do Castelo (55.30\%).

Respecto a los servicios de comunicación que presenta los indicadores globales relativos a los sistemas de interacción, demuestran un peso del 64,97\% relativo a distintos servicios de comunicación de los ciudadanos con los ayuntamientos, aunque el peso varía por distritos. El promedio más alto ha sido para Lisboa (79,2\%), seguido del distrito de Portalegre (71,1\%), y el menor para el distrito de Beja (23,83\%).

Es significativo que al estudiar los servicios de transacción (vida cotidiana) que contempla la reserva o compra de billetes para eventos deportivos y culturales se alcance la cifra del 70,45\%. Por distritos es en Setúbal donde se alcanza el porcentaje medio más alto (76,9\%), seguido por el distrito de Bragança (70,85\%). Mientras el resultado más bajo lo ofrece el Distrito de Faro (3,1\%).

\subsubsection{Administración a distancia}

Los indicadores de los distintos servicios relacionados con la administración a distancia manifiestan resultados dispares. Así, por un lado, se presentan valores porcentuales elevados para los servicios de información con un 94,94\%, y para los servicios de transacción con un 83,63\% para el total de Portugal; y, por otro, los servicios de comunicación reflejan unas cifras muy inferiores, de sólo el 25,95\%, lo que parece indicar una menor implicación de 
los cuadros administrativos con los ciudadanos a través de sistemas de comunicación tan simples como el correo electrónico.

Respecto a los distritos, estos son los resultados en el ámbito general de la administración a distancia:

- Relativo a los servicios de información el porcentaje promedio más alto lo presenta el distrito de las Azores (97,80\%), seguido por el distrito de Évora (94,04\%) y el más bajo para el distrito de Viana do Castelo (75\%).

- En los servicios de comunicación, el porcentaje medio más alto ha sido para el distrito de Lisboa (79,2\%), seguido por el distrito de Viseu (66,7\%) y el más bajo para el distrito de Aveiro (5,3\%).

- Y sobre los servicios de transacción, el porcentaje más alto lo tiene el distrito de Viseu (89,27\%), seguido del distrito de Bragança (86,91\%), y el valor más bajo es para la Comunidad autónoma de Azores (23,33\%).

\subsubsection{Participación política}

El apartado relacionado con los servicios de información es el que tiene más vínculos con la gestión de los contenidos, en tanto que contempla la existencia de actas de plenos de los ayuntamientos, decretos de los alcaldes, etc. Los porcentajes aquí son los más elevados de todo el estudio (99,9\%) para el conjunto de Portugal. Muchos de los distritos alcanzan el 100\%, como Beja, Bragança, Évora, Faro, Lisboa, Portalegre y Vila Real, siendo el valor más bajo para el distrito de las Azores con el 91,23\%.

Muy diferentes son los resultados para los indicadores relativos a los servicios de comunicación y de transacción. Así, el peso de los servicios de comunicación con el ámbito político apenas representa un 11,3\%, mientras que no existe ningún sistema de votación ciudadana en el periodo de realización del estudio. Aunque en los servicios de comunicación dos distritos destacan sobre la media, la Región autónoma de Azores con un 33,7\%, seguido por el distrito de Setúbal con un peso del 32,28\%.

\section{CONCLUSIONES}

Globalmente el estudio sobre los contenidos en los 308 ayuntamientos de Portugal define una administración local avanzada, en la línea del esfuerzo realizado en Portugal en la administración central, aunque en el momento de realización del trabajo de campo Portugal no tuviera aprobada una ley de transparencia, como sí la tienen un importante número de países europeos.

Respecto a los indicadores evaluados y que ha estructurado el trabajo de investigación en tres tipos de servicios (servicios de información, servicios de comunicación y servicios de transacción) aplicados a tres ámbitos bien diferenciados como son los aspectos de la vida cotidiana, los aspectos referidos a la vida administrativa de las corporaciones locales y los aspectos relativos a la vida política de estas administraciones los resultados han sido dispares.

Respecto a los servicios de información, el volcado de información -de todo tipo- en los portales de las administraciones públicas en internet, ha conformado la fase inicial de la administración electrónica en todo el mundo. La facilidad en desarrollar un sistema en el que la información como materia, con una mayor o menor elaboración, estuviera a disposición de los ciudadanos ha facilitado el establecimiento de estos servicios de información a través de los portales de una manera rápida. En este estudio los servicios de información presentan porcentajes muy elevados (por encima del 90\%) en los ámbitos de "administración a distancia” y "participación política”, lo que implica una aceptación, por parte del poder local de la necesidad de acercarse a los ciudadanos; y elevados en el ámbito de vida cotidiana, en torno a un $70 \%$ de los indicadores examinados.

Por el contrario, los servicios de comunicación presentan resultados diversos. Mientras están más desarrolladas las herramientas para interactuar en el ámbito de la vida cotidiana, la posibilidad de interactuar de manera bilateral con funcionarios o políticos tiene menos peso, probablemente como resultado de la ausencia de una ley de transparencia y buen gobierno. Finalmente los servicios de transacción muestran un comportamiento dispar. Así, mientras las opciones que permiten realizar algún tipo de transacción tienen porcentajes muy elevados en el ámbito de la vida cotidiana (64,97\%) y en el ámbito de la administración a distancia (83,63\%), lo que facilita la vida de los ciudadanos al hacer realidad la máxima de la administración a distancia respecto al espacio y al tiempo -el espacio que los ciudadanos no tienen que recorrer hasta los edificios públicos y el consiguiente ahorro de tiempo-, la posibilidad de realizar votaciones por internet es inexistente. 


\begin{abstract}
NOTAS
${ }^{1}$ Resumen de la tesis doctoral: La información electrónica das câmaras municipales portuguesas. Tesis doctoral realizada por Eduardo Cardoso Miranda en el Departamento de Información y Comunicación de la Universidad de Extremadura (España). Enero de 2012.

${ }^{2}$ En este contexto de transformación y de reingeniería de los procesos de las Administraciones, los profesionales de la gestión de la información y la documentación presentes en ámbitos tradicionales como los archivos o las bibliotecas, se encuentran ahora con un nuevo ámbito en el que poder desarrollar su trabajo. Dicho de otro modo, la aplicación de las TICs a las organizaciones requiere de profesionales informáticos pero también de profesionales de la gestión de la información que pueden encargarse de aspectos muy diversos, como la arquitectura de la información, el diseño web, la edición de contenidos o la usabilidad de la información, entre otras habilidades y competencias.

3 Son los siguientes: teletrabajo, educación a distancia, red de universidades y centros de investigación, servicios telemáticos para las Pymes, gestión del tráfico por carretera, gestión del tráfico aéreo, redes de asistencia sanitaria, licitación electrónica, red transeuropea de administraciones públicas y autopistas urbanas de la información.

${ }^{4}$ Se hace necesario reflejar el proceso de evolución y expansión de los medios sociales y en particular de las redes sociales para contextualizar la razón por la que no se incluyeron en la etapa de recogida entre 2009 y comienzos de 2010. La consultora española de investigación de mercados The Cocktail Analysis, especializada en tendencias de consumo y nuevas tecnologías, señala varias fases en la etapa evolutiva de las redes sociales: una etapa inicial de 2008 a 2009 como fenómeno emergente que busca de manera generalizada tener presencia, experimentar y probar. Una etapa de consolidación de 2009 a 2010 en las que aparece un discurso normalizado debido a que las redes sociales se convierten en una herramienta de comunicación generalizada, si bien en un contexto de cambio donde unas redes aparecen y desaparecen, y otras se consolidan. Y una fase, de 2010 a 2011, de expansión donde las redes sociales llegan a la casi totalidad de internautas y no son territorio exclusivo de los usuarios debido a la presencia de las marcas. En esta etapa se produce también el salto a la movilidad, a través de la telefonía móvil. The Cockatil Analysis [en línea]. Disponible en: <http://tcanalysis.com> [Consulta: 10 de diciembre de 2012].
\end{abstract}

\title{
7. BIBLIOGRAFÍA
}

ALLEN, A.B.; JUILLET, L.; PAQUET, G. y ROY, J. E-Governance and government online in Canada: partnerships, people and prospects. Government Information Quarterly, 2001, vol. 1, nº 2, p. 93-104.

ANMP - Associação Nacional de Municípios Portugueses. Disponible en: <http://www.anmp.pt> [Consulta: 20 de noviembre de 2012].

APDSI - Associação para a Promoção e Desenvolvimento da Sociedade da Informação. Glossário Sociedade da Informação [en línea]. Disponible en: <http://www.apdsi.pt> [Consulta: 2 de octubre de 2011].

ARMSTRONG, C.L. Providing a clearer view: An examination of transparency on local government websites. Government Information Quarterly, 2011, vol. 28, nº 1, p. 11-16.

BERTOT, J.C. y JAEGER, P.T. The E-Government paradox: Better customer service doesn't necessarily cost less. Government Information Quarterly, 2008, vol. 25, n 2, p. 149-154.

BRANCO, F. Municípios e Políticas Sociais em Portugal 1977-1989. Lisboa: Instituto Superior de Serviço Social, 1998.

CASTELlS, M. y HIMANEN, P. A Sociedade da Informação e o Estado-Providência: O Modelo Finlandês. Lisboa: Fundação Calouste Gulbenkian, 2007.

CHAIN-NAVARRO, C.; MUÑOZ-CAÑAVATE, A. y SALIDO MARTÍNEZ, V. LIS education and web services in the public sector: the case of Spain. Libri, 2008, vol. 58, n 4, p. 246-256.

CHAIN-NAVARRO, C.; MUÑOZ-CAÑAVATE, A. y MÁS BLEDA, A. La gestión de información en las sedes web de los ayuntamientos españoles. Revista Española de Documentación Científica, 2008, vol. 31, nº 4, p. 612-638.

CHAN, C.M.L; LAU, Y. y PAN, S.L. E-government implementation: A macro analysis of Singapore's e-government initiatives. Government Information Quarterly, 2008, vol. 25, nº 2, p. 239-255.

CHEN, A.; PAN, S.L.; ZHANG, J.; WEI HUANG, W. y ZHU, S. Managing e-government implementation in China: A process perspective. Information \& Management, 2009, vol. 46, nº 4, p. 203-212.

CLEVELAND, H. Government is Information (but Not ViceVersa). Public Administration Review, 1986, vol. 46, p. 605-607.

COMISIÓN EUROPEA. Libro Blanco. Crecimiento, competitividad y empleo. Retos y pistas para entrar en el siglo XXI. COM (93) 700 final.

COMISIÓN EUROPEA. Europa en marcha hacia la sociedad de la información. COM (94) 347 final.

COMISIÓN EUROPEA. La información del sector público: un recurso clave para Europa. Libro Verde sobre la información del sector público en la sociedad de la información. COM (1998) 585.

COURSEY, D. y NORRIS, D.F. Models of e-government: Are they correct? An empirical assessment. Public Administration Review, 2008, vol. 68, n 3, p. 523-536.

DUARTE DOS SANTOS, L. y MARTINS DO AMARAL, L.A. A Presença das Câmaras Municipais Portuguesas na internet. Guimarães: Universidade do Minho, 2000.

GIL-GARCÍA, J.R. y MARTÍNEZ-MOYANO, I.J. Understanding the evolution of e-government: The influence of systems of rules on public sector dynamics. Government Information Quarterly, 2007, vol. 24, n 2, p. $266-290$. 
GOUVEIA, L.B. y TEIXEIRA, P. Local e-government: A situação das juntas de Freguesia do concelho de Vila Nova de Gaia [en línea]. Disponible en: <http://www2.ufp.pt/ lmbg/com/rev_ufp05_egovl.pdf> [Consulta: 2 de octubre de 2011].

GROZNIK, A. y TRKMAN, P. Upstream supply chain management in e-government: The case of Slovenia. Government Information Quarterly, 2009, vol. 26, n 3, p. 459-467.

HOLLIDAY, I. y YEP, R. E-government in China. Public Administration and Development, 2005, vol. 25, $\mathrm{n}^{\circ}$ 3, p. 239-249.

LAYNE, K. y LEE, J. Developing fully functional E-government: a four stages model. Government Information Quarterly, 2000, vol. 18, n 2, p. 122-136.

Livro Verde para a Sociedade da Informação em Portugal [en línea]. Disponible en: <http://www.missao-si.mct.pt> [Consulta: 1 de mayo de 2010].

MACEDO E SOUSA, L. Onde as Coisas Acontecem: Comunicação, Sociedade, Poder, Administração Pública. Lisboa: Hugin Editores, Lda., 2000.

MUÑOZ-CAÑAVATE, A. La Administración General del Estado en internet. Un estudio sobre la VI Legislatura. Badajoz: UEX, 2003.

MUÑOZ-CAÑAVATE, A. Administración electrónica ¿e-ciudadanos o e-burocracia? Ágora. El debate peninsular. Cáceres: Junta de Extremadura, 2006, p. 63-87.

MUÑOZ-CAÑAVATE, A. y CHAÍN-NAVARRO, C. La Administración local española en internet (1997-2002). Murcia: Servicio de Publicaciones de la UMU, 2012.

MUÑOZ-CAÑAVATE, A. y HIPOLA RUIZ, P. Electronic Administration in Spain. Government Information Quarterly, 2011, vol. 28, n 1, p. 74-90.

OCDE. The e-Government Imperative. OECD Publishing, 2003.

PEDROSO, A. A Administração Electrónica em Portugal. Estimular a inclusão digital, a eficiência da Administração e o crescimento sócio-económico. Ponencia presentada en el curso “Administración Electrónica ¿e-ciudadanos o eburocracia?” dentro de las Jornadas Ágora. Cáceres (España), 25 de octubre de 2006.

SHI, Y. The accessibility of Chinese local government Web sites: An exploratory study. Government Information Quarterly, 2007, vol. 24, n 2, p. 377-403.

UMIC - Agência para a Sociedade do Conhecimento: Ministério da Ciência, Tecnologia e Ensino Superior [en línea]. Disponible en: <http://www.umic.pt> [Consulta: 2 de octubre de 2011].

VIANA de SÁ, L. Introdução ao Direito das Autarquias Locais. Lisboa: Universidade Aberta, 2000. 


\section{ANEXO. INDICADORES OBTENIDOS DE LAS NUEVE ÁREAS DE ESTUDIO}

Indicadores obtenidos en el ámbito de vida cotidiana

\begin{tabular}{|c|c|c|c|}
\hline Ámbito general & \multicolumn{3}{|c|}{ Vida cotidiana } \\
\hline Áreas de estudio & Servicios de información & Servicios de comunicación & $\begin{array}{l}\text { Servicios de } \\
\text { transacción }\end{array}$ \\
\hline $\begin{array}{l}\text { Puntuación } \\
\text { aplicada }\end{array}$ & $0-2$ & $0-1$ & $0-1$ \\
\hline \multirow[t]{11}{*}{ Indicadores } & $\begin{array}{l}\text { Información general } \\
\text { - Datos postales de la ciudad } \\
\text { - Información de la historia de la ciudad } \\
\text { - Información turística (monumentos) } \\
\text { - Teléfonos de interés del municipio }\end{array}$ & \multirow[t]{11}{*}{$\begin{array}{l}\text { Foros de discusión } \\
\text { - Lista de correo electrónico } \\
\text { - A través de foros } \\
\text { - A través de chat }\end{array}$} & \multirow[t]{11}{*}{$\begin{array}{l}\text { Reserva o compra } \\
\text { de billetes } \\
\text { - Teatro municipal } \\
\text { - Eventos } \\
\text { deportivos }\end{array}$} \\
\hline & $\begin{array}{l}\text { Información sobre salud pública } \\
\text { - Sistema sanitario (instalaciones) } \\
\text { - Horario de las farmacias }\end{array}$ & & \\
\hline & $\begin{array}{l}\text { Información sobre educación } \\
\text { - Instalaciones } \\
\text { - Oferta escolar } \\
\text { - Bolsas de estudio } \\
\end{array}$ & & \\
\hline & $\begin{array}{l}\text { Información sobre deporte } \\
\text { - Instalaciones deportivas } \\
\text { - Eventos deportivos (agenda) }\end{array}$ & & \\
\hline & $\begin{array}{l}\text { Información sobre cultura } \\
\text { - Museos } \\
\text { - Biblioteca municipal } \\
\text { - Otras bibliotecas } \\
\text { - Eventos culturales } \\
\text { - Consulta online } \\
\end{array}$ & & \\
\hline & $\begin{array}{l}\text { Información sobre los servicios sociales } \\
\text { - Instalaciones para mujeres } \\
\text { - Instalaciones para jóvenes } \\
\text { - instalaciones para inmigrantes }\end{array}$ & & \\
\hline & $\begin{array}{l}\text { Información sobre transportes } \\
\text { - Autobuses } \\
\text { - Metro } \\
\text { - Avión } \\
\text { - Tren } \\
\text { - Marítimo } \\
\text { - Horarios }\end{array}$ & & \\
\hline & $\begin{array}{l}\text { Información urbana } \\
\text { - Mapas de la ciudad } \\
\text { - Mapas interactivos de la ciudad }\end{array}$ & & \\
\hline & $\begin{array}{l}\text { Información estadística } \\
\text { - Sobre la población } \\
\text { - Sobre datos económicos } \\
\end{array}$ & & \\
\hline & $\begin{array}{l}\text { Información comercial } \\
\text { - Sobre mercados } \\
\text { - Sobre comercio }\end{array}$ & & \\
\hline & $\begin{array}{l}\text { Información sobre seguridad } \\
\text { - Policía (contactos) } \\
\text { - Bomberos (contactos) } \\
\text { - Protección civil (contactos) }\end{array}$ & & \\
\hline
\end{tabular}


Indicadores obtenidos en el ámbito de Administración a distancia

\begin{tabular}{|c|c|c|c|}
\hline Ámbito general & \multicolumn{3}{|c|}{ Administración a distancia } \\
\hline Áreas de estudio & Servicios de información & Servicios de comunicación & Servicios de transacción \\
\hline $\begin{array}{l}\text { Puntuación } \\
\text { aplicada }\end{array}$ & $0-1$ & 0-1 & $0-1$ \\
\hline \multirow[t]{7}{*}{ Indicadores } & $\begin{array}{l}\text { Directorio de los servicios } \\
\text { del ayuntamiento } \\
\text { - Teléfono } \\
\text { - Correo electrónico }\end{array}$ & \multirow{7}{*}{ 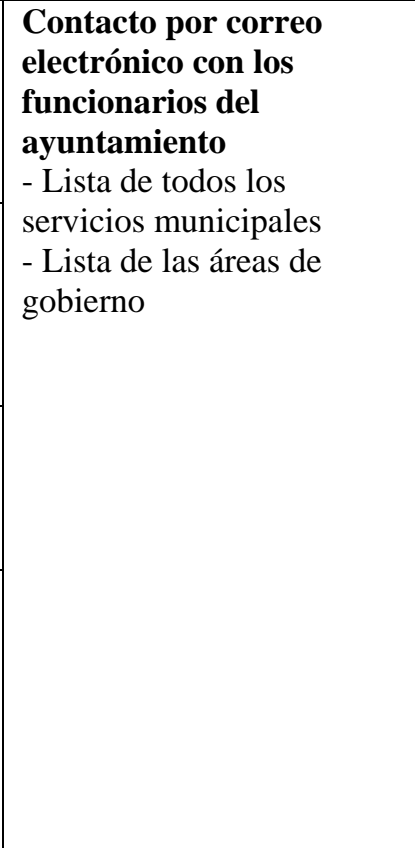 } & $\begin{array}{l}\text { Presentación electrónica } \\
\text { documentos/solicitudes/trámites } \\
\text { población } \\
\text { - Recepción de documentos }\end{array}$ \\
\hline & - Correo postal & & \multirow{2}{*}{$\begin{array}{l}\text { Educación } \\
\text { - Recepción de documentos }\end{array}$} \\
\hline & Trámites administrativos & & \\
\hline & $\begin{array}{l}\text { - Guía de trámites } \\
\text { administrativos (cómo hacer) } \\
\text { - Ordenanzas fiscales } \\
\text { - Ordenanzas municipales }\end{array}$ & & $\begin{array}{l}\text { Territorio, urbanismo y obras } \\
\text { - Trámites de expedientes de } \\
\text { obras } \\
\text { - Consulta de expedientes }\end{array}$ \\
\hline & $\begin{array}{l}\text { Servicios de prensa } \\
\text { - Comunicados } \\
\text { - Agenda de los actos del }\end{array}$ & & $\begin{array}{l}\text { Circulación, vehículos y } \\
\text { transportes } \\
\text { - Pago de impuesto de vehículos }\end{array}$ \\
\hline & alcalde & & \multirow[b]{2}{*}{$\begin{array}{l}\text { Comercio, industria y consumo } \\
\text { - Trámites sobre la apertura de } \\
\text { establecimientos } \\
\text { - Estado del expediente sobre } \\
\text { licencias/actividad }\end{array}$} \\
\hline & $\begin{array}{l}\text { Asamblea municipal } \\
\text { - Nombre del alcalde } \\
\text { - Correo electrónico del } \\
\text { alcalde } \\
\text { - Nombre de los concejales } \\
\text { - Correo electrónico de los } \\
\text { concejales }\end{array}$ & & \\
\hline
\end{tabular}

Indicadores obtenidos en el ámbito de participación política

\begin{tabular}{|l|l|l|l|}
\hline Ámbito general & \multicolumn{3}{|c|}{ Participación política } \\
\hline Áreas de estudio & $\begin{array}{l}\text { Servicios de } \\
\text { información }\end{array}$ & $\begin{array}{l}\text { Servicios de } \\
\text { comunicación }\end{array}$ & Servicios de transacción \\
\hline Puntuación aplicada & $0-2$ & $0-1$ & $0-1$ \\
\hline Indicadores & $\begin{array}{l}\text { Documentos } \\
\text { - Actas de plenos } \\
\text { - Boletín municipal de } \\
\text { información } \\
\text { - Decretos y/o despachos } \\
\text { del alcalde }\end{array}$ & $\begin{array}{l}\text { Debates de problemas } \\
\text { políticos }\end{array}$ & $\begin{array}{l}\text { Votación por internet } \\
\text { - Votación }\end{array}$ \\
& & & \\
\hline
\end{tabular}

